\title{
Kernos
}

Revue internationale et pluridisciplinaire de religion grecque antique

$5 \mid 1992$

Varia

\section{Le vin, la mort et les Bienheureux (à propos des lamelles orphiques)}

\section{Maria de Henar Velasco López}

\section{OpenEdition \\ Journals}

Édition électronique

URL : http://journals.openedition.org/kernos/1061

DOI : 10.4000/kernos. 1061

ISSN : 2034-7871

Éditeur

Centre international d'étude de la religion grecque antique

Édition imprimée

Date de publication : 1 janvier 1992

ISSN : 0776-3824

Référence électronique

Maria de Henar Velasco López, « Le vin, la mort et les Bienheureux (à propos des lamelles orphiques) », Kernos [En ligne], 5 | 1992, mis en ligne le 19 avril 2011, consulté le 01 mai 2019. URL http://journals.openedition.org/kernos/1061; DOI : 10.4000/kernos.1061 
Kernos, 5 (1992), p. 209-220.

\section{LE VIN, LA MORT ET LES BIENHEUREUX (À PROPOS DES LAMELLES ORPHIQUES)}

Les deux lamelles d'or, trouvées à Petroporos en $1985^{1}$, furent une surprise pour les chercheurs de ce domaine pour de nombreuses raisons :

1) Elles furent découvertes en un lieu assez éloigné des autres lieux de découverte de lamelles d'or ${ }^{2}$; dans cette région, on connaît seulement la lamelle thessalienne ${ }^{3}$ et une autre trouvée à Pharsale.

2) Elles se présentent sous une forme particulière : au lieu d'être rectangulaires comme les autres, elles ont été découpées en forme de cœur ou de feuille de lierre.

3) Elles constituent le trait d'union entre deux séries différentes de lamelles d'or : celles de Mnémosynè et celles de Thourioi.

4) Elles confirment l'existence de la "formule du chevreau» des tablettes de Thourioi avec d'importantes variantes.

5) Elles témoignent de la liaison de ces documents avec Dionysos, puisqu'au second vers, nous lisons Báxıos.

6) Elles fournissent une référence claire au liquide de prédilection de ce dieu, le vin qui, jusqu'à la découverte de ces tablettes, restait inconnu des lamelles d'or. Y apparaissaient uniquement, l'eau de Mnémosynè que le défunt prie d'apaiser sa soif, et le lait dans lequel tombe le chevreau, ou selon le témoignage des lamelles de Petroporos, le taureau ou le bouc ${ }^{4}$.

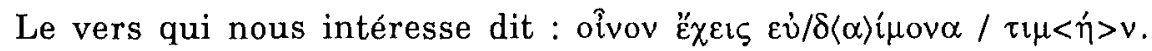
C'est le dernier vers de la lamelle «b» de Petroporos, tandis qu'à la lamelle «a», c'est l'avant-dernier, immédiatement suivi de la promesse que le défunt, qui ce même jour est mort, est revenu à la vie. Il doit

1 K. Tsantsanoglou - G.M. Parassoglou, Two Gold Lamellae from Thessaly, in Hellenika, 38 (1987), p. 3-16.

2 G. ZunTz, Persephone. Three Essays on Religion and Thought in Magna Grecia, Oxford, 1971, p. 275-393.

3 J. BresLin, A Greek Prayer, Ambassador College, Pasadena, 1977.

4 Ces trois liquides apparaissent dans une image orphico-pythagoricienne transmise par PlatoN, Gorgias, 493d-e : l'homme ordonné a ses tonneaux pleins de ces liquides (du vin, du lait, du miel, etc.) et il ne s'en soucie plus, mais l'homme dissolu, comme les siens sont troués et pourris, doit les remplir sans cesse. Cf. aussi à propos des rapports entre ces liquides : HoR., Carm., II, 19, 10; Ioel., III, 18; CLAUDIEN, De raptu Proserpinae, II, 351 sq. 
communiquer à Perséphone que Bacchus l'a libéré et l'attendent sous la

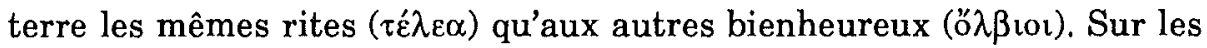
deux lamelles, le vers apparaît après les trois formules de la chute dans le lait. À la suite de la rupture de rythme, que celles-ci introduisent, on attendrait le retour au mètre dactylique, mais dans ce cas, il s'agit d'un tetramètre, le seul exemple dans l'ensemble des lamelles. Il se peut que, comme les heptamètres qui apparaissent parfois dans les textes des tablettes, le rédacteur soit le responsable, capable de garder le rythme dactylique, mais sans plus créer des hexamètres corrects.

Si l'on a tellement insisté sur la place du vers dans le texte, c'est parce qu'à notre avis, la liaison avec le contexte fournit une des clés pour son interprétation. En premier lieu, la lecture de Tsansanoglou et Parasoglou ne nous semble pas convaincante. Ces auteurs ${ }^{5}$ proposent de

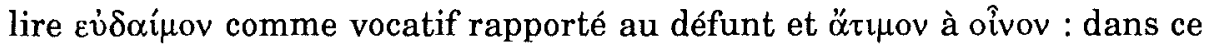
cas, le vin serait sans paiement, libre de charges, ce qu'ils justifient par le caractère proberbial de l'abondance de la richesse dans l'Hadès. Mais, comme ils le reconnaissent eux-mêmes, $« \alpha \tau \tau \mu(\mu) \nu$ may not be

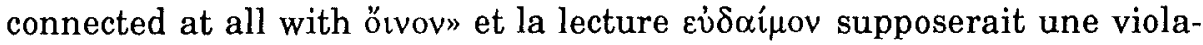
tion du zeugma de Hermann. De plus, tant dans cette lamelle que dans les autres, l'épithète généralement employée pour faire allusion au

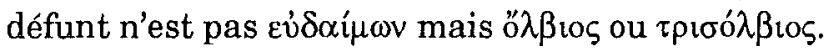

Par conséquent, nous pensons que la lecture doit être $\varepsilon \dot{\delta} \delta \alpha i \mu o v \alpha \tau \imath \mu \eta ́ v$, en tant qu'apposition à oîvov, que l'on qualifie d'«heureuse récompense». De plus, le verbe est au présent, ع̌ $\chi \varepsilon 1 \zeta$, ce qui introduit un changement

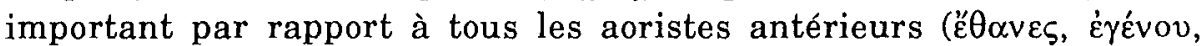

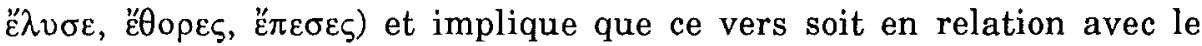

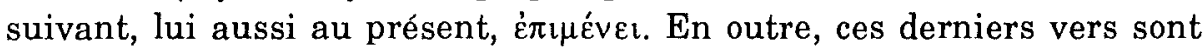

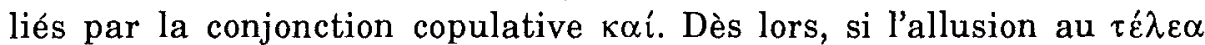
qui attendent le défunt fait référence à un prix que l'on promet ímò $\gamma \hat{\eta} v$,

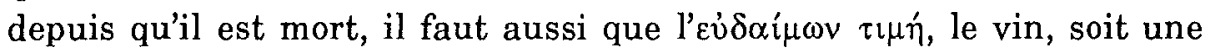
récompense dont il profite dans l'au-delà.

Le rédacteur change donc la perspective temporelle en parlant au défunt au présent, comme s'il bénéficiait déjà de ces faveurs. Nous ne pouvons pas oublier que les deux phrases suivantes sont des formules où le taureau et le bouc tombent dans le lait, et, que l'on considère celles-ci comme l'écho d'un rituel ou comme des formules gnomiques, elles constituent des mots de passe, qui supposent que le défunt a déjà atteint le comble du bonheur et, comme le premier vers le dit, qu'il n'est pas mort mais vivant. Ces deux derniers vers se plaisent à louer et à décrire les

5 Loc. cit., p. 14. 
dons que le défunt obtiendra dans l'autre monde. La lamelle «b», qui finit par ce vers, confirme le fait; dans cette lamelle, les vers 4 et 7 de «a» ne sont pas présents, parce qu'ils ne sont pas nécessaires, plutôt que pour des raisons d'espace, puisque les autres vers donnent déjà une information suffisante. La promesse du vin est suffisante pour combler les désirs du défunt, sans qu'il soit nécessaire de mentionner les $\tau e ́ \lambda \varepsilon \alpha$.

Donc, seul l'examen du texte de la tablette nous permet d'envisager, avec une idée plus claire, les propositions que Tsansanoglou et Parasoglou font pour ce vers : il ferait allusion aux libations funéraires données au mort, ou au vin que l'initié a goûté en tant que participant aux mystueres de Dionysos, ou au vin dont il entend profiter dans l'audelà. L'analyse interne des vers montre que cette dernière hypothèse est la plus vraisemblable, bien que, comme nous verrons plus loin, nous ne puissions pas la détacher de la pratique des libations et, d'une certaine façon, les promesses de l'au-delà trouvent leur correspondance dans les rites que le défunt a célébrés de son vivant, comme les $\tau e ́ t \lambda \varepsilon \alpha$ du vers suivant. Comme, dans les autres lamelles, on fait appel à la vision des prairies et des forêts de Perséphone en tant que lieux charmants pour les défunts $^{6}$, ou à la promesse d'un royaume partagé ${ }^{7}$, ici aussi l'image du bonheur dans l'autre monde a pu être façonnée selon les activités que, de son vivant, le défunt a préférées en tant qu'initié.

Outre l'analyse interne du texte, nous disposons d'autres témoignages littéraires qui décrivent les délices de l'autre monde comme un banquet où l'on ne peut manquer de vin.

Dans l'Iliade, une expression singulière signifie que les dieux ont

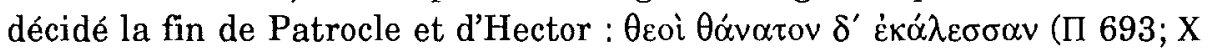
297). On fait usage de $\kappa \alpha \lambda \varepsilon \hat{v} v$ pour inviter un hôte à un repas ${ }^{8}$. Déjà Eustathe $(1081,53)$ considère l'expression comme une $\sigma \varepsilon \mu v \eta ́$ $\pi \varepsilon p i \varphi p \alpha \sigma i \varsigma$ pour mourir. Si l'expression est la même, nous ne pouvons que nous demander, avec Vermeule, s'il ne s'agit pas d'une invitation au banquet parce que le banquet est une des images courantes de l'au-delà.

Ce n'est pas une référence isolée. Pindare (Ném., I, 69) présente Héraclès, qui est le paradigme d'une vie vertueuse qui reçoit le prix de

6 Lamelle 4 de Thourioi v. 6, dans la terminologie de Zuntz A4.

7 Lamelle de Pétélia v. 11, dans la terminologie de Zuntz B1.

$8 \quad \delta 532 ; \rho 52 ; \lambda 187 ;$ o $213 ; \lambda 410 ; \kappa 231=257=313$. Cf. H. EBELING, Lexicon Homericum, Leipzig, 1885 (réimpr. Hildesheim, 1963), s.v. к $\alpha \lambda \varepsilon \hat{\imath} v$. E. Vermeule, Aspects of Death in Early Greek Art and Poetry, Berkeley, 1979, trad. espagnole México, 1984, p. 79, n. 65, remarque aussi qu'on l'utilise pour une invitation au lit ( $\beta 348$ ), à la maison ( $\lambda 410)$, à la conversation séparée (o 529). 
l'immortalité, participant à un banquet auprès de Zeus, même s'il s'agit du banquet nuptial que l'on célèbre au ciel pour ses noces avec Hébé.

Des poètes postérieurs adoptent aussi ce motif pour rapprocher le sort du héros de ce qui attend d'autres personnages humains. Ainsi Théocrite ${ }^{9}$ place-t-il Ptolémée dans la demeure de Zeus, assis sur un trône d'or, en face de la stalle d'Héraclès, et célébrant des festins avec les autres dieux; et il ajoute qu'Héraclès, rassasié de nectar parfumé, quitte le banquet pour aller près de son épouse. Horace ${ }^{10}$ offre la même image en rapport avec Auguste, qu'il représente couché entre Pollux et Héraclès, "purpureo bibit ore nectar».

Si, dans ces cas, la boisson dont profite Héraclès, Ptolémée ou Auguste est le nectar, c'est parce que le banquet se célèbre avec les dieux, dont le nectar est la boisson préférée. Mais chez Horace ${ }^{11}$, nous trouvons une référence différente : après avoir parlé de la participation d'Héraclès aux banquets de Jupiter, le poème finit sur ces vers : ornatus viridi tempora pampino / Liber vota bonos ducit ad exitus. Bien que l'allusion de Liber = Bacchus soit très dépouillée, elle ne laisse pas d'être significative et nous permet d'enchaîner avec les autres textes où l'on imagine les défunts bienheureux célébrant un banquet, et buvant, non du nectar, parce que leur demeure, bien que fortunée, n'est pas la même que celle des dieux, mais du vin.

De courtes références aux banquets que les défunts célèbrent dans

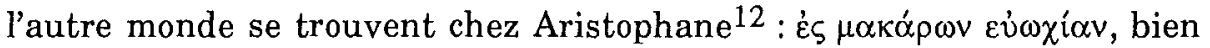

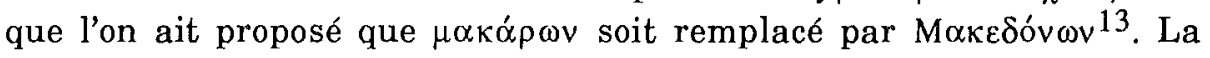
description de tels festins, avec beaucoup de détails, se retrouvent dans les fragments d'autres auteurs comiques, comme Aristophon, Phérécrate et Téléclides.

Un des éléments communs est la référence à la boisson. Aristophane lui-même, dans les T $\alpha \gamma \eta v \imath \sigma \alpha i^{14}$, fait allusion à l'obligation de boire après être descendu dans l'autre monde, bien qu'il ne précise pas s'il s'agit de vin ou d'un autre liquide; mais, puisqu'il en fait la cause de

9 Idylle, XVII, 15 sq.

10 Carmina, III, 3, 10 sq.

11 Carmina, IV, 8, 33 sq.

12 Ran., v. 85; cf., avec plus de références au banquet post mortem que celles qui ont été soulignées ici et la bibliographie, F. GRAF, Eleusis und die orphische Dichtung Athens in vorhellenistischer Zeit, Berlin-New York, 1974, p. 98-103.

13 Cf. éd. Stanford, New York, $1968^{2}$.

14 Fr. 504, 8 Kassel-Austin (Poetae Comici Graeci, Berolini et Novi Eboraci, 1983 1989). 
l'expression $\mu \alpha \kappa \alpha ́$ por attribuée aux défunts, il serait difficile de la comprendre s'ils buvaient de l'eau. Aristophon (fr. 12), précisément, se moque des Pythagoriciens qui mangent là-bas des légumes et boivent de l'eau, c'est-à-dire persévèrent dans leur diète terrestre. À notre avis, l'affirmation que leur situation est très différente ( $\delta 1 \alpha \varphi e^{\prime} \rho \varepsilon v \nu \delta \dot{\varepsilon} \pi \alpha \dot{\alpha} \mu \pi \mathrm{o} \lambda v$, v. 2) de celle des autres défunts et l'épithète ev̉xepńs (v. 5) que l'on attribue à Hadès parce qu'il accepte de manger avec eux et boit de l'eau en leur honneur ( que les autres bienheureux boivent du vin et se régalent, comme l'attestent les autres auteurs comiques.

Phérécrate, évoquant la richesse proverbiale d'Hadès (cf. l'épithète Ploutôn), décrit notamment dans le fragment 113 la manière dont des jeunes filles, comme de nouvelles Danaïdes, offrent des coupes pleines

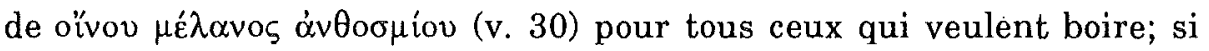
quelqu'un en veut encore, il y en a deux fois plus. Dans le fragment 137 du même auteur, c'est Zeus qui fait pleuvoir le vin (v. 6), en arrosant abondamment, de telle sorte que le liquide et d'autres mets sont recueillis depuis les toits.

Chez Téléclides (fr. 1), les fleuves de jus noir qui entraînent toutes sortes de gâteaux, comme chez Phérécrate (fr. 113 v. 3; fr. 117, v. 3), sont

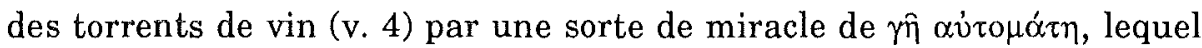
correspondrait aux meilleures descriptions de l'âge d'or ${ }^{15}$ ou aux moments les plus exaltés des rituels bachiques ${ }^{16}$.

En outre, à ces témoignages littéraires, nous pouvons ajouter un témoignage artistique. Il s'agit d'un vase italique qui représente Dionysos accomplissant un miracle du vin : sans intervention humaine, le vin coule des grappes de raisin dans les coupes ${ }^{17}$. Cette représentation est notamment intéressante parce que, derrière l'amphore, le miracle est répété et, dans ce cas, à la place de Dionysos, il y a un mortel, certainement un initié. M. Schmidt, bien que l'idée d'y voir une représentation des bienheureux à l'Elysée soit tentante, suppose qu'il s'agit plutôt de ceux qui de leur vivant sont destinés au bonheur,

15 Hes., Op., 117 sq.; HoR., Ep., 16, 44.

16 SopH., Athamas, fr. 5 P.; Eur., Ba., 142; 704-711; cf. commentaire dans l'édition de E.R. Dodds (Oxford, 1944) aux vers 704-711, et J. Roux (Paris, 1970-1972); Eur., Hyps., fr. 57 (cf. commentaire de G.V. Bond, Oxford, 1963 [réimpr, avec corrections 1969, p. 119 sq.]).

17 Cf. M. SCHMIDT, Orfeo e orfismo nella pittura vascolare italiota, in Orfismo in Magna Grecia. Atti del quattordicessimo Convegno di Studi sulla Magna Grecia. Taranto 6-10 ottobre 1974, Napoli, 1975, p. 105-137, p. 134 sq., pl. XVI. 
parce que la participation aux mystères de Dionysos comporte sur la terre un état presque paradisiaque.

Or, si pour figurer le bonheur sur terre, on utilise une image de l'audelà, c'est parce qu'existe la conviction ferme que tel sera le destin qui attend les adeptes de Dionysos. Il nous semble que cette image doit être mise en rapport avec les textes déjà mentionnés à propos des mets qui attendent les défunts dans l'autre monde, de même qu'avec le vers commenté des lamelles de Petroporos. De plus, le vase qui porte cette représentation a été découvert dans une tombe où l'on a également trouvé une représentation d'Orphée et d'un défunt avec un rouleau à la main, peut-être une sorte de passeport pour l'au-delà, semblable aux lamelles d'or ${ }^{18}$. Schmidt n'écarte pas l'idée que le défunt enseveli dans ce tombeau ait appartenu à une secte dionysiaque influencée par des idées orphiques, ou plutôt à une religion orphico-dionysiaque ${ }^{19}$.

Dans tous les témoignages examinés, le vin comme aliment promis aux bienheureux est abondant, facile à obtenir, et aucun d'entre eux ne fait allusion à l'ivresse éternelle que Platon censure dans sa République (363c). Prêtant ces théories à Musée et à son fils, il décrit un banquet auquel participent les őoıol, c'est-à-dire les initiés aux cérémonies orphiques ou mystai. Sa réticence à l'égard de cette récompense de la vertu en tant que $\mu \varepsilon \dot{\theta} \theta \eta v \alpha_{i \omega ́} \iota_{\text {ov }}{ }^{20}$ doit peut-être être comprise dans le même sens que ses critiques aux orpheotelestai, comme une remarque à l'encontre des exagérations et des erreurs que pouvaient engendrer ces doctrines ${ }^{21}$.

18 Cf. loc. cit., p. 113 sq.; cf. p. 230; pl. VIII.

19 Loc. cit., p. 134. Dans l'hymne à Dionysos (v. 35 sq.), le dieu fait un miracle semblable : quand il est monté comme un prisonnier à bord d'un navire, il commence à faire couler du vin. Pour d'autres miracles en rapport avec le vin, cf. H. Jeanmaire, Dionysos. Histoire du culte de Bacchus, Paris, 1951, p. 24; M.P. NILSSON, Griechische Feste von religiöser Bedeutung mit Ausschluss der Attischen, Stuttgart, 1906 (réimpr. Darmstadt, 1957), p. 292 sq.; R. Merkelbach, Die Hirten des Dionysos. Die Dionysos-Mysterien der römischen Kaiserzeit und der bukolischen Roman des Longus, Stuttgart, 1988, p. 54 sq.

20 La même expression est reprise par PluTARQUE, Luc., 44, 2, qui cite Platon et rapporte lui aussi telle croyance aux fidèles d'Orphée.

21 Dans le même sens, P.M. ScHUHL, Essai sur la formation de la pensée grecque, Paris, 1934, p. 238. Sur de possibles échos de l'idée du banquet lié à l'ivresse, différent du banquet eucharistique (PAUL, I Cor., 11, 21), et leur représentation chez les premiers chrétiens, cf. R. EISLER, Orphisch-dionysische Mysteriengedanken in der christlichen Antike, Hildesheim, 1966, p. 174 et n. 5. 
Il ne s'agit pas d'un état d'ivresse perpétuel, mais parmi le mets du banquet qui attend les défunts dans l'au-delà, le vin est présent, comme il est présent dans les banquets sur terre ${ }^{22}$. Il ne faut pas oublier que, dans des doctrines ésotériques, l'accès à l'ivresse spirituelle, qui cause l'oubli de soi-même et engendre la vraie connaissance, commence par une ébriété physique. Celle-ci est par exemple évoquée sans aucune nuance péjorative à la fête irlandaise de Samain, pendant laquelle, grâce à l'ivresse, les hommes croient s'approcher du monde des dieux ${ }^{23}$.

Même si la boisson préférée des dieux est le nectar ${ }^{24}$, cependant Onians ${ }^{25}$ soutient que le nectar est l'équivalent divin du vin considéré comme une forme d'aliment convenable pour les dieux, et il allègue beaucoup de textes à l'appui de son hypothèse. Nous avons déjà remarqué que, si Héraclès boit du nectar, c'est parce que sa place est parmi les dieux, tandis que les autres défunts, bien qu'ils attendent un sort meilleur que les obscures profondeurs d'Hadès, ne partagent pas leur demeure avec les dieux.

Le vin est une boisson propre aux bienheureux parce qu'il était la boisson habituelle sur terre, du moins le vin mélangé, mais surtout parce que le vin est par excellence la liqueur de Dionysos, et nous ne pouvons pas oublier que, dans le second vers des lamelles de Petroporos, on lit : "dis à Perséphone que Bacchus t’a libéré/e».

Nous ne pouvons nous étendre ici sur la discussion de Dionysos comme dieu du vin, ni sur la plus ou moins grande ancienneté de cette caractéristique de sa personnalitét ${ }^{26}$. Nous voulons, par contre, souli-

22 J. HARRISON, Prolegomena to the Study of Greek Religion, Cambridge, $1959^{3}$ [1903], p. 614, défend l'idée que livresse éternelle était entendue, parmi les Orphiques les plus savants, comme une extase spirituelle, tandis que les moins instruits la considéraient comme un banquet mérité. On aurait popularisé cette notion par le biais de la croyance au banquet des héros que les fidèles de Dionysos auraient adoptée. Nous pouvons remarquer que Platon lui-même (Ax., 371d) fait allusion à l'existence de banquets dans l'au-delà, sans qu'il y ait dans ce cas aucune nuance péjorative, bien au contraire.

23 Cf. J. Chevalier - A. Gheerbrant, Dictionnaire des symboles, Paris, 1969 (version espagnole, Barcelona, 1986) s.v. ébriété; G. DuRAND, Les structures anthropologiques de l'imaginaire, Paris, 1963 (trad. espagnole, Madrid, 1981), p. 248 sq., avec une bibliographie en rapport avec les peuples indo-européens.

24 Mais on peut voir l'estime à l'égard du vin chez les dieux : O. KE RN, Orphicorum Fragmenta, Berlin, 1922, fr. 297.

25 R.B. ONIANS, The Origins of European Thought about the Body, the Mind, the Soul, the World, Time and Fate, Cambridge, 1954, p. 296-299.

26 En plus des ouvrages de Burkert et de Nilsson, cf. par exemple : HARrison, op. cit., p. 415 sq., p. 517. Elle pense que Dionysos est avant tout le dieu d'une 
gner qu'il existe des indices permettant d'affirmer que boire du vin dans ses rites n'était pas un acte de pur divertissement, mais une sorte de sacrement solennel, au cours duquel le vin remplace le sang de Dionysos et joue le rôle de liqueur d'immortalité ${ }^{27}$, même si certains, comme Cicéron ${ }^{28}$, considéraient le fait de donner au vin le nom de Liber n'était qu'une figure de rhétorique et que personne ne pouvait être fou au point de croire qu'il était en train de boire le dieu quand il portait une coupe à ses lèvres.

Le vin est le symbole de la connaissance de l'initiation dans les traditions sémitiques. Il est, même, la boisson des dieux; la vigne ellemême est un arbre sacré, qui épouse parfois l'iconographie de l'arbre de la vie et, en conséquence, le vin est le symbole de la jeunesse et de la vie éternelle.

céréale intoxicante, dont l'écho se retrouve dans des épithètes comme Bromios, Braites, Sabazios, qui supposeraient l'existence d'une boisson utilisée parmi les peuples du nord. Á son avis, ces boissons spiritueuses faites avec des grains fermentés auraient remplacé l'hydromel et, ensuite, elles auraient été remplacées par le vin (à propos du remplacement par le vin d'autres boissons plus anciennes, cf. I. CHIRASSI, Elementi di culture precereali nei miti e riti greci, Roma, 1968, p. 36 sq., p. 82 sq.); JEANMAIRE, op. cit., p. 22-35 considère que Dionysos, avant de présider à la culture de la vigne, a été un dieu de la vigne sauvage, et que, chez les Grecs, il n'est pas reçu comme génie du vin, mais comme génie de la végétation.

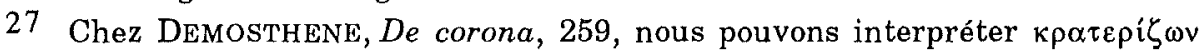
comme le geste des initiés versant le vin mystique, le sang de DionysosZagreus tué par les Titans. Cf. NILsson, op. cit., p. 260 sq.; ONIANS, op. cit., p. 278, n. 2; W. BURKert, Homo Necans, Berlin, 1972 (trad. anglaise Univ. California, 1983, p. 224 sq. et p. 246). À propos de la signification de oîvo set de

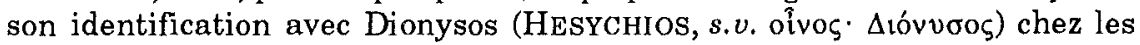
Orphiques, cf. O. KERN, op. cit., n. 213, 216, 240 et 241; cf. aussi H. USENER, Göttliche Synonyme, in RhM, 53 (1898), p. 329-379, surtout p. 375 (= Kleine Schriften, Leipzig-Berlin, 1913, p. 259-306, surtout p. 303); O. HöFER, art Oinos, in Roscher, III, 1 (1908), p. 799; J. MURR, Die Pflanzenwelt in der Griechischen Mythologie, 1890 (réimpr. Gröningen, 1969), p. 135; EISLER, op. cit., p. 178-186, après avoir souligné que boire du vin pour les Orphiques est la même chose que recevoir Dionysos, le $i \lambda_{\text {Lkò }}$ vov̂s, il poursuit avec la référence à MACrobe, in Somn. Scip., I, $12=\mathrm{KERN}, 421$, sur la demeure des âmes

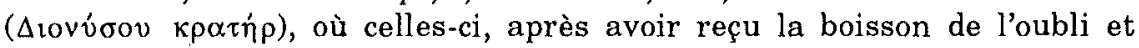
l'ivresse (le vin), tombent à travers les sphères du ciel jusqu'au tombeau et la prison du corps terrestre. Il se peut que ce passage de Macrobe soit une interprétation tardive qui combine les idées de vin = oubli et vin = aliment de l'autre monde, avec la croyance à la transmigration des âmes et, en conséquence, qu'il utilise le vin comme remplaçant de l'eau de Léthé, qui mène à l'oubli.

28 De natura deorum, III, 41. 
Dans la tradition grecque également des proverbes et des jeux de mots mettent en rapport le vin et l'intelligence :

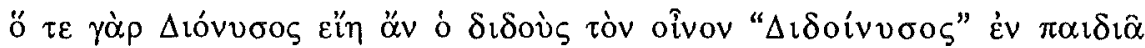

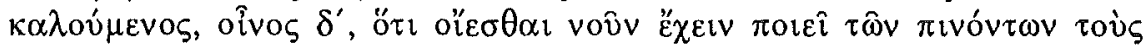

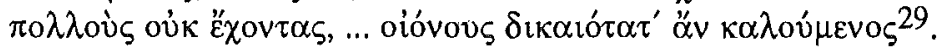

de même que le vin et la vérité, et des expressions semblables apparais-

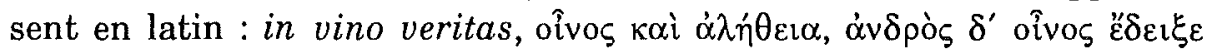
vóov 30 .

Il existe également des relations non négligeables entre le vin et la mort. Quelques exemples suffiront. Les Anthestéries, tout d'abord, faisaient coïncider les rites que supposent la désacralisation du vin de la dernière récolte, dont les prémices étaient offertes au dieu au début du printemps $^{31}$, et le caractère funeste du jour du triomphe de Dionysos puisque les âmes des morts revenaient le temps d'une journée sur la terre pour visiter leurs parents. L'ambiguité des Anthestéries se retrouve dans la parenté entre les $\chi$ oés, nom du premier jour de la fête pendant lequel se tenait un concours dont le but était d'avaler le plus rapidement possible le vin contenu dans ces cruches, et les $\chi o \alpha i$, libations du dernier jour en l'honneur des morts.

En second lieu, de nombreuses références associent le vin à la folie ou à la mort, chez Homère ${ }^{32}$ comme chez d'autres auteurs grecs ${ }^{33}$. Les Grecs se distinguent des Barbares en buvant du vin mélangé et, conformément au proverbe delphique, $\mu \eta \delta \dot{\varepsilon} v \not \alpha \gamma \alpha v$, avec mesure ${ }^{34}$. Ils

29 Platon, Crat., 406c; cf. le commentaire de Proclos, 182.

30 ATHENEE, II, 35 sq.

31 Dans quelques traditions locales, les cérémonies supposent que le dieu apparaisse comme émissaire de l'au-delà, entre les marais, bouches des enfers. Cf. Nilsson, op. cit., p. 267-271 et Jeanmaire, op. cit., p. 48-56 avec bibliographie à la page 486; BURKERT, op. cit., p. 213-226.

$32 \lambda$ 291-304; $\varphi$ 61-65. Cf. à ce propos E.R. DoDDs, The Greeks and the Irrational,

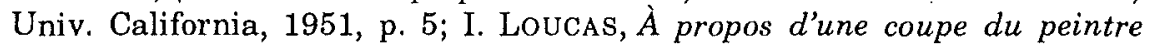
d'Amasis, in $A C, 53$ (1984), p. 270-273; ONIANS, op. cit., p. 33-36, p. 42 sq., p. 152.

33 HDT., VI, 84, 1; ATHENEE, II, 36b. La même relation est présente aussi chez d'autres peuples indo-européens. Ainsi chez les Celtes, sont fréquentes les disputes qui commencent par l'ivresse (H. D'ARBoIs De JubaInville, Cours de littérature celtique, I, p. 297) et, dans le Mahabharata, on trouve des références au jeu qui monte à la tête comme le vin.

34 Cf. ATHENEE, II, 37b. 
méprisent l'ivresse que produit le vin pur chez les Barbares, qui, quand ils connaissent cette boisson ${ }^{35}$, la consomment sans la mélanger ${ }^{36}$.

C'est pur, sans mélange, contrairement à l'usage des banquets, que les Grecs utilisent le vin pour les libations funéraires. Graf ${ }^{37}$, qui a étudié la présence de différents liquides dans les libations, pense que la libation sans vin n'est pas le vestige d'un ancien rituel remontant à une époque où le vin était inconnu, puisque le terme oîvos et les formes véhiculées dans d'autres langues indo-européennes sont attestés à haute époque $^{38}$. La présence de l'un ou l'autre liquide répond, à son avis, à une fonction définie : les libations avec vin mélangé, qui sont les plus courantes, supposent une liaison avec l'ici et le maintenant, tandis que celles de vin pur apparaissent dans des contextes marginaux, notamment quand on cherche le contact avec l'au-delà et les morts ${ }^{39}$.

On comprend ainsi la moquerie de Lucien 40 affirmant que, bien que les parents versent du vin pur sur la tombe, celui-ci n'arrivera pas à l'Hadès, où les défunts ont leurs propres aliments ${ }^{41}$, de même que l'utilisation de vin pur par Ulysse pour attirer les âmes 42 . Le vin mélangé est un symbole de civilisation; le vin pur, cause de la mort du Cyclope et d'autres géants ${ }^{43}$, apporte la folie et la mort aux hommes, et s'intègre dans un usage marginal, mais très important dans les libations funéraires. Considéré comme une boisson propre aux peuples étrangers que l'on méprise, le vin pur se retrouve néanmoins au pays des Bienheureux; le vin est un élixir de vie ${ }^{44}$ ou la vie même, comme dit Trimalcion : "vita vinum est» 45 . Pensons encore à la fête romaine des Anna Perenna au cours de laquelle on demande aux dieux d'accorder

35 HDT., IV, 60, 2, pour les Scythes; PLuT., Is., 6, pour les Égyptiens.

36 HDT., VI, 84, 1, pour les Scythes; ATHENEE, I, 34b, pour les Égyptiens.

37 F. GRAF, Milch, Honig und Wein. Zum Verständnis der Libation im griechischen Ritual, in Perennitas. Studi in onore di A. Brelich, Roma, 1980, p. 209-221.

38 Cf. P. Chantraine, Dictionnaire étymologique de la langue grecque, Paris, 1968-1980, s.v. ổvos.

39 Cf. BuRKERT, op. cit., p. 54 sq.

40 De luctu, 19.

41 Pour la présence du vin sur les tombes à inscriptions funéraires, cf. R. LATTIMORE, Themes in Greek and Latin Epitaphs, Urbana, 1962, p. 128, 133.

$42 \kappa 519=\lambda 27$.

43 VERMEULE, op. cit., p. 233, cf. p. 309.

44 DURAND, op. cit., p. 248.

45 PeTr, Sat., 34, 7; cf. W. DEONNA - M. RENARD, Croyances et superstitions de table dans la Rome antique, Bruxelles, 1961, p. 32 sq. 
autant d'années que de coupes de vin consommées ${ }^{46}$; pensons également aux saluts écrits sur les coupes : "Vivas", "Bibe multos annos",

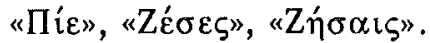

Onians ${ }^{47}$ pense que la raison de l'identification du vin et de la vie provient de la relation entre l'homme et les plantes; ce liquide serait pour l'homme mort ce que la sève est pour les végétaux et c'est la raison pour laquelle l'homme mort, sec d'avoir soif, a besoin d'un liquide qui contienne la vie et la force. Ce liquide, dans beaucoup de traditions, est l'eau; sur les lamelles d'Hippone, de Pétélia, de Pharsale, de Thessalie et de Crète le défunt demande de l'eau qui coule de la lagune de Mnémosnynè, une eau qui préserve de l'oubli. Le but des libations est justement de perpétuer la vie, et dans les rites funéraires, le vin joue un rôle très important : les os du cadavre incinéré étaient lavés avec du vin $^{48}$, le vin est présent au banquet funéraire ${ }^{49}$, et certains, comme Trimalcion, souhaitent que, même parmi les représentations de sa tombe, figure une amphore avec du vin 50 .

On connaît la coïncidence, soulignée par Onians 51 , entre les offrandes que reçoivent les dieux (du vin et de la graisse) et celles qui, chez Homère, apparaissent sur les os et les cendres des morts.

46 OvIDE, Fasti, III, 523 sq. Il faut remarquer qu'au retour de la fête ceux qui y ont participé sont nommés fortunatos (v. 539).

47 Op. cit., p. 215 sq.

48 ONIANS, op. cit., p. 282; $\Psi 220$ sq. et $250 ; \Omega 791$ sq.; PETR., Sat., 65; 77, 7.

49 PETr., Sat., 78, 3. Le banquet funéraire, auquel on pense aussi que prend part le défunt (E. RoHDE, Psyche. Seelenkult und Unsterblichkeitsglaube der Griechen, 1894, version espagnole México, 1948, p. 113), est aussi attesté en Thrace (HDT., V, 8), et dans leurs célébrations XENOPHON, Hell., III, 2, 5, dit que l'on avale du vin. Chez les Égyptiens, on trouve la même coutume : les serviteurs redoublent de zèle pour offrir les coupes de vin : «bois, jusqu'à ce que tu sois ivren; une dame réclame davantage de vin, car tout en elle n'est que paille; chez une autre, l'ébriété est plus forte, assise par terre, elle se répand en lamentations (A. ERMANN, Die Religion der Ägypter, Berlin, 1934, trad. franç. Paris, 1937, p. 278). Nous pouvons nous demander si la consommation exagérée du vin aux banquets funéraires de la Thrace et des Égyptiens n'est pas liée au fait que telle boisson conduit à l'oubli (ATHENEE, II, 35c). Peut-être cherche-t-on aussi une imitation du sort du défunt dans l'au-delà, puisque, dans la tradition égyptienne, quoique la boisson préférée des défunts soit la bière, nous trouvons dans les textes des pyramides : «sa nourriture est au milieu des dieux et son eau est du vin comme celle de Rề (HERMANN, op. cit., p. 253).

50 PETR., Sat., 71.

51 Op. cit., p. 282 sq. 
Cet auteur indique aussi que cette assimilation entre l'élément de la vie et un liquide, l'eau ou le vin, explique que l'affranchissement d'un esclave se termine lorsqu'il boit un de ces liquides en disant : «j'ai bu l'eau de la libertés 52 . Dionysos, lui-même, dieu du vin, reçoit le titre de

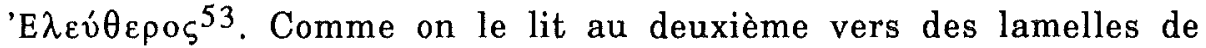
Pétroporos, Dionysos a libéré le défunt de la vie, et maintenant commence la nouvelle vie dans laquelle il pourra profiter du vin qui l'attend comme récompense.

Departemento de Filología Clásica

\section{Maria de Henar VELASCO LOPEZ}

Facultad de Filosofía y Letras

Universidad de Valladolid

E - 47002 VALLADOLID

52 ANTIPH., fr. 25; cf. XeNARCH., fr. 5; Z 528 sq. Cf. ONANS, op. cit., p. 479.

53 On peut voir dans EISLER, op. cit., p. 119 sq. un aspect différent de celui des rapports entre l'action libératrice et le vin dans le culte dionysiaque. 